Supporting Information

\title{
Analysis of bacteriophage-host interaction by Raman tweezers
}

Zdeněk Pilát ${ }^{1 *}$, Alexandr Jonáš ${ }^{1}$, Jana Pilátová ${ }^{2}$, Tereza Klementová ${ }^{1}$, Silvie Bernatová ${ }^{1}$, Martin Šiler ${ }^{1}$, Tadeáš Maňka ${ }^{1}$, Martin Kizovskýl, Filip Růžička ${ }^{3}$, Roman Pantůček ${ }^{4}$, Ute Neugebauer ${ }^{5,6}$, Ota Samek ${ }^{1}$, Pavel Zemánek ${ }^{1}$

${ }^{1}$ Institute of Scientific Instruments of the Czech Academy of Sciences, v.v.i., Czech Academy of Sciences, Královopolská 147, 61264 Brno, Czech Republic

${ }^{2}$ Department of Experimental Plant Biology, Faculty of Science, Charles University, Viničná 5, 128 44 Prague 2, Czech Republic

3 Department of Microbiology, Faculty of Medicine, Masaryk University and St. Anne's Faculty Hospital, Pekařská 53, 65691 Brno, Czech Republic

${ }^{4}$ Department of Experimental Biology, Faculty of Science, Masaryk University, Kotlářská 2, 61137 Brno, Czech Republic

${ }^{5}$ Center for Sepsis Control and Care (CSCC), Jena University Hospital, Am Klinikum 1, D-07747 Jena, Germany

${ }^{6}$ Leibniz Institute of Photonic Technology (Leibniz-IPHT), Albert-Einstein-Str. 9, D-07745 Jena, Germany

Corresponding author:pilat@isibrno.cz

\section{Contents}

Supplementary Figure S1

page S2

Supplementary Figure S2

page S3

Supplementary Figure S3

page S4 


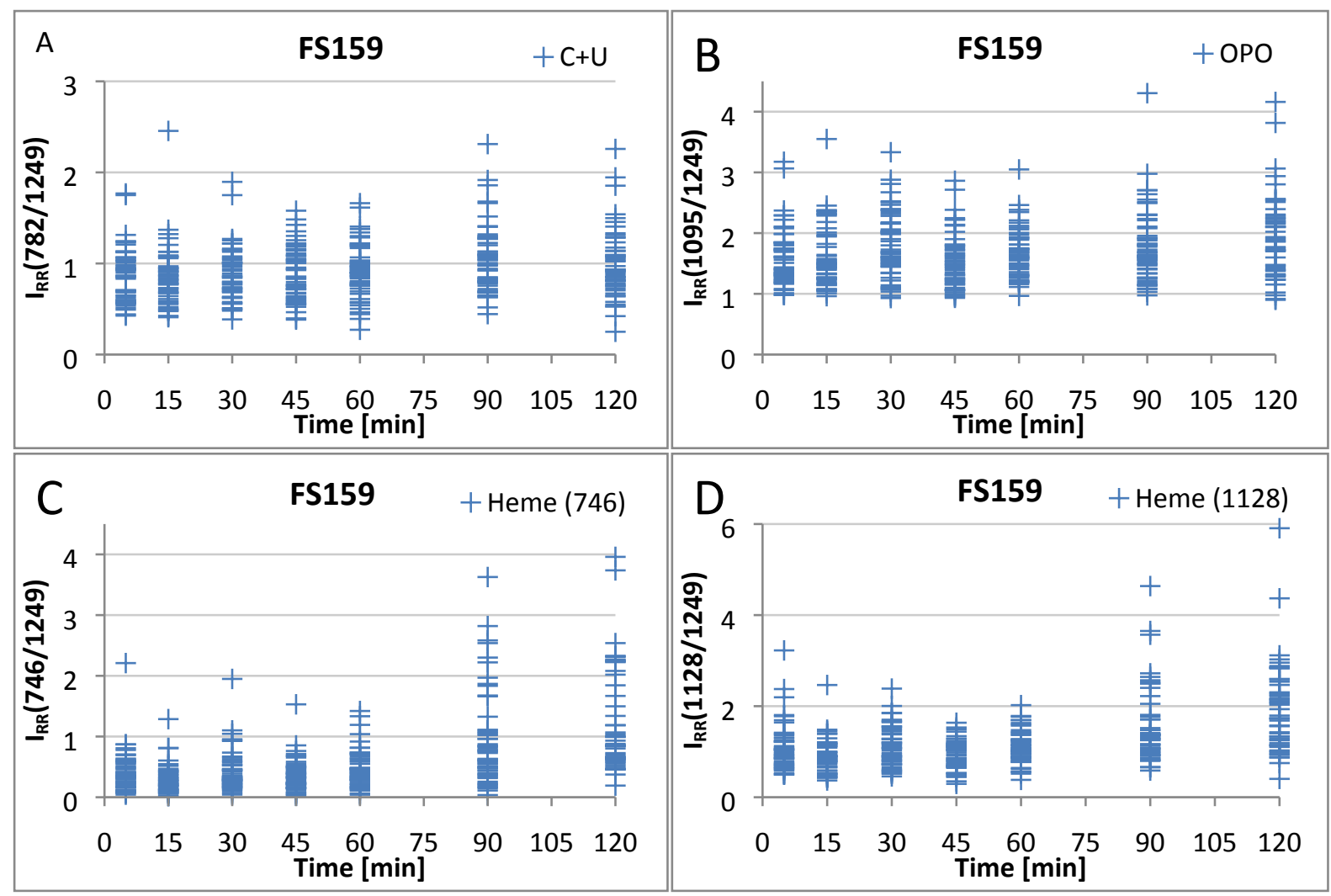

Figure S1. Scatter plots representing individual measurements of selected Raman spectral ratios $I_{R R}$ during the cultivation of control host cells FS159. The numerical values of $I_{R R}$ in each graph represent the intensities of Raman spectral peaks of interest normalized to the spectral peak of protein-specific vibration of amide bond Am III. The actual wavenumbers of Raman peaks involved in calculating $\mathrm{I}_{\mathrm{RR}}$ values are listed in $\mathrm{cm}^{-1}$ in the plot labels. a) Ratio $\mathrm{C}+\mathrm{U} / \mathrm{Am}$ III represents the vibration of nucleic bases cytosine and uracil. b) Ratio OPO/Am III represents the vibration of phosphodiester bond in nucleic acids and phospholipids. $\mathbf{c}+\mathbf{d})$ Ratios Heme/Am III represent two different molecular vibrations of heme, a cofactor for respiratory enzymes. 


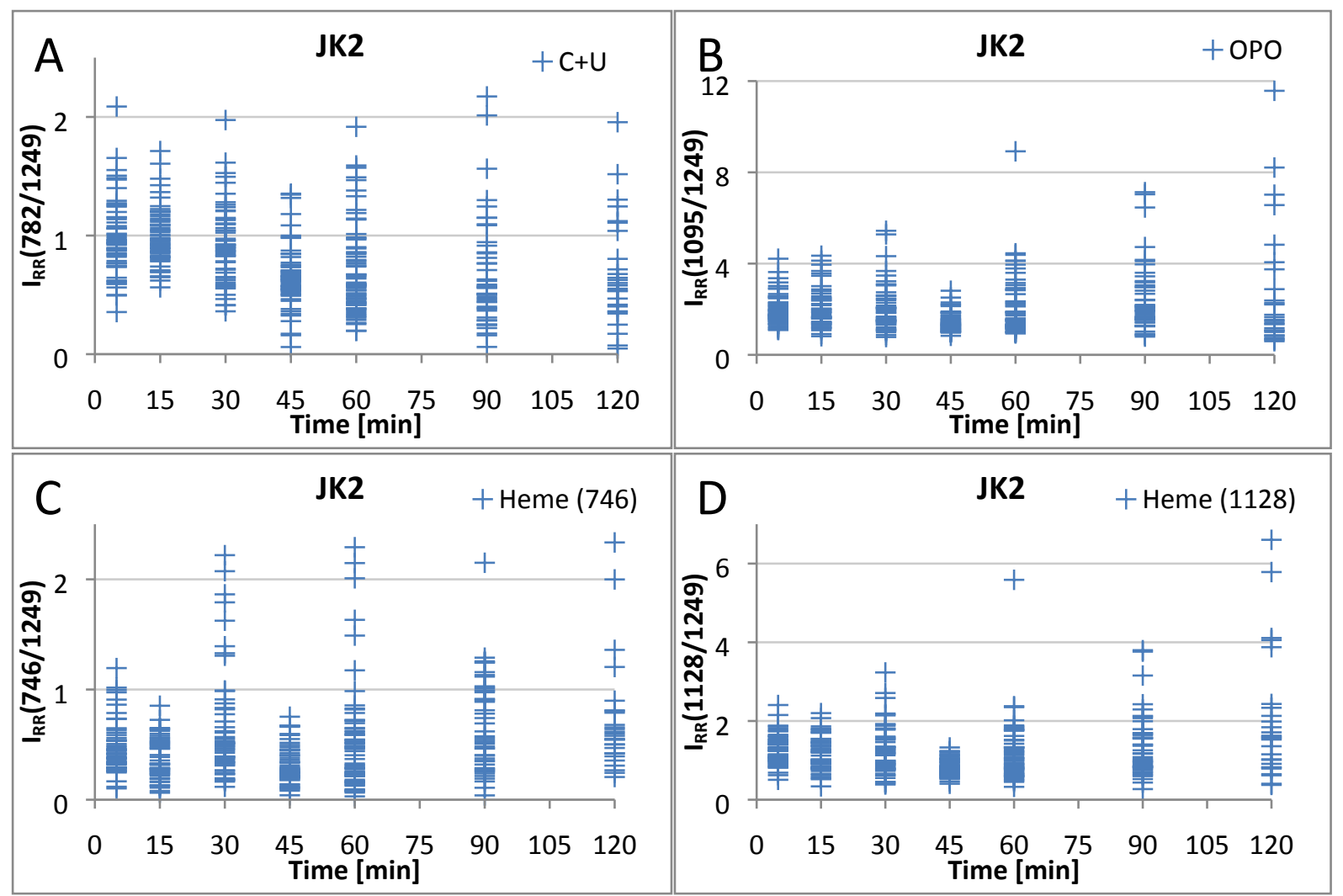

Figure S2. Scatter plots representing individual measurements of selected Raman spectral ratios $I_{R R}$ during the cultivation of JK2 phage-infected FS159 cell cultures. The numerical values of $I_{R R}$ in each graph represent the intensities of Raman spectral peaks of interest normalized to the spectral peak of protein-specific vibration of amide bond Am III. The actual wavenumbers of Raman peaks involved in calculating $\mathrm{I}_{R R}$ values are listed in $\mathrm{cm}^{-1}$ in the plot labels. a) Ratio $\mathrm{C}+\mathrm{U} / \mathrm{Am}$ III represents the vibration of nucleic bases cytosine and uracil. b) Ratio OPO/Am III represents the vibration of phosphodiester bond in nucleic acids and phospholipids. $\mathbf{c}+\mathbf{d})$ Ratios Heme/Am III represent two different molecular vibrations of heme, a cofactor for respiratory enzymes. 


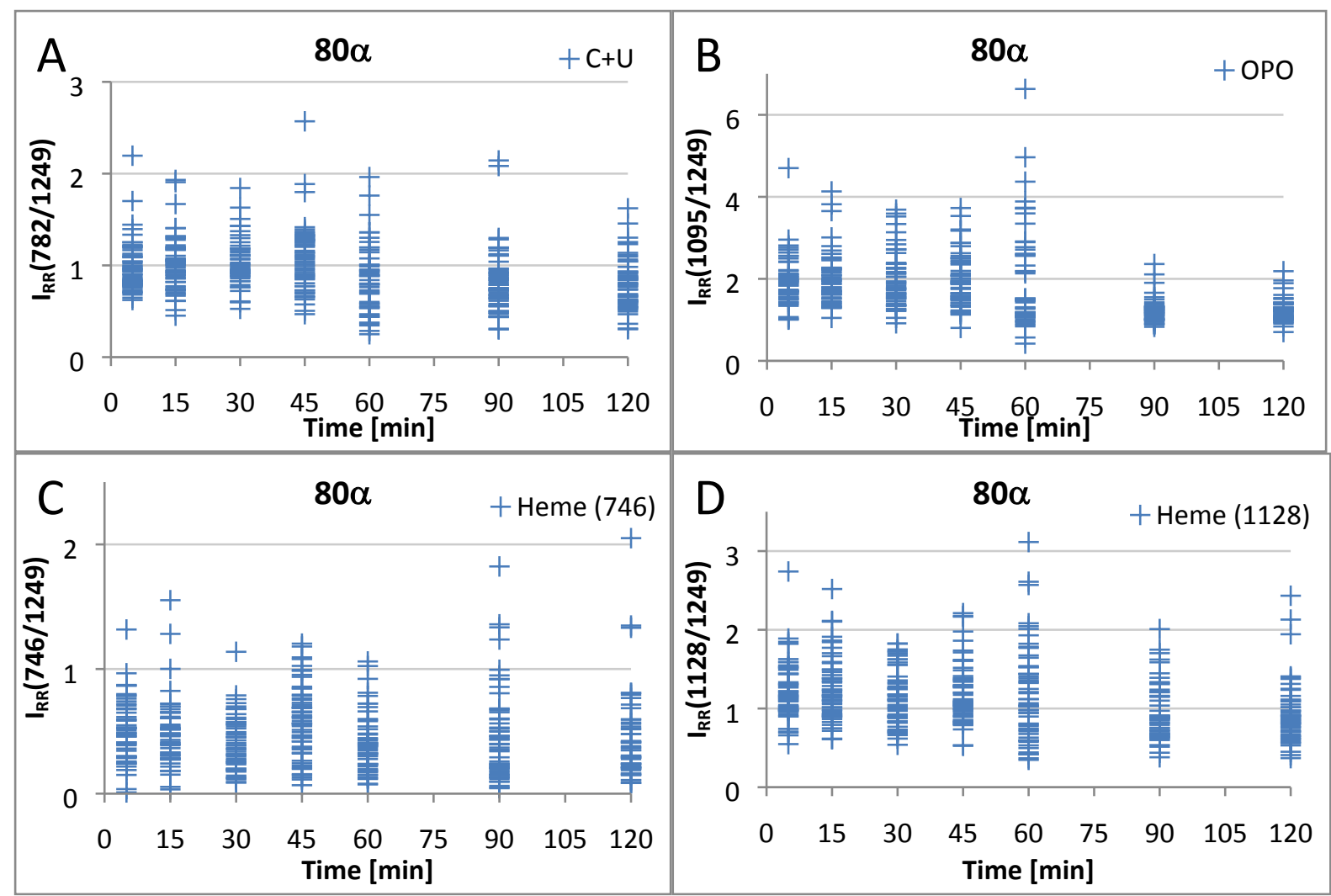

Figure S3. Scatter plots representing individual measurements of selected Raman spectral ratios $I_{R R}$ during the cultivation of $80 \alpha$ phage-infected FS159 cell cultures. The numerical values of $I_{R R}$ in each graph represent the intensities of Raman spectral peaks of interest normalized to the spectral peak of protein-specific vibration of amide bond Am III. The actual wavenumbers of Raman peaks involved in calculating $I_{R R}$ values are listed in $\mathrm{cm}^{-1}$ in the plot labels. a) Ratio C+U/Am III represents the vibration of nucleic bases cytosine and uracil. b) Ratio OPO/Am III represents the vibration of phosphodiester bond in nucleic acids and phospholipids. $\mathbf{c}+\mathbf{d})$ Ratios Heme/Am III represent two different molecular vibrations of heme, a cofactor for respiratory enzymes. 